\title{
Risk-based assessment of climate change impact on storm drainage system
}

\author{
Z. Alsaqqaf, H. Zhang \& S. Mohamed \\ Griffith School of Engineering, Griffith University, Australia
}

\begin{abstract}
Climate change impacts on engineering infrastructures are increasing. The infrastructures are expected to withstand more frequent and severe weather events, more climate variability, and changes in climate norms (average conditions). It is anticipated that many civil infrastructure systems such as storm drainage, will fail to meet the expected environmental pressures. Therefore, it is important to identify current and future risks; to develop strategies for adapting such risks; and to implement an effective maintenance plan. In the study, the climate change impacts on storm drainage were investigated, particularly in Southport, Queensland, Australia. The historical rainfall data for 120 years were analysed to identify the changes in the trends, patterns and frequencies of rainfall. The peak flow in a flooding event was identified. The investigation provides essential information for the vulnerability to risk failure of the existing storm drainage system, such as at a critical pipe failure point. Finally, the research applied a risk-based vulnerability assessment by risk analysis and management quantification tools to quantify the impact that rainfall may induce through storm drainage failure.
\end{abstract}

Keywords: storm drainage, climate change, risk assessment, event tree analysis.

\section{Introduction}

Due to increased and more intense Climate Change (CC), engineering structures are increasingly forced to withstand more frequent and extreme weather events, more climate variability, and changes in climate norms (average conditions). Numerous changes have already occurred, such as the increase in the global mean temperature and the sea level rise. These changes, projected to worsen over time, will impact on civil infrastructure systems, such as storm drainage, in 
a number of ways. Therefore, a detailed investigation is needed to identify those risks, identify how to overcome them, and identify how to implement management of infrastructure maintenance planning that copes with future projected CC risks.

This study examines $\mathrm{CC}$ impacts, especially precipitation rates in relation to storm drainage planning and design. The research introduces $\mathrm{CC}$ variables that may affect storm drainage and identifies the critical risks involved. Engineering risk management is discussed, along with a justification for the impacts from the anticipated precipitation rates. In addition, it justifies the impacts from the probability of precipitation rates, and a review of the impacts on existing storm drainage in Southport, Queensland, Australia. Moreover, this research applies the risk management approach by quantifying the vulnerability of storm drainage to rainfall. It then provides a brief of risk adaptation theories, risk identification, and the decision making tools and techniques required. Some of the limitations of the research as a whole and the model used are addressed. These limitations were due to a mix of time constrains and a shortage of data. However, the limitations were minimised through scientific and logical assumptions. Finally, the research depicts adaptation guidelines with implications for engineers and designers. The paper provides engineers and decision makers with essential information regarding the vulnerability risk of storm drainage cased by $\mathrm{CC}$, as represented by rainfall and flooding events. While such risks are accepted under certain conditions, according to their management strategies, this is not always so. This study provides designers and planners ways to adjust or reassess the risk, and thus reach the most feasible and acceptable level of risk. In other words, engineers and decision makers will be able to set a benchmark for damage probabilities to achieve the safest outcomes in the most economic way.

\section{Infrastructure risk analysis}

The aim of this paper is to measure the impact on the storm drainage by intense rainfall. These measurements are achieved by, firstly analysing the hydrological conditions through an examination of the historical rainfall data probabilities of the rainfall events for a storm drainage case study at a defined location. Secondly, the research produced probabilities of the storm drainage failure. This was achieved by pipe and hydrology calculations which will be represented later in this study. Thirdly, the two probabilities are combined to generate the failure of the storm drainage in a defined rainfall event.

The design of this study incorporated two models. Firstly, the whole research context adopted the Infrastructure Risk Analysis Model (IRAM); which was developed by Ezell [3]. The second model was the Neural Network Deterioration Model (NNDM), which is specialized in assessing the deterioration of the storm drainage systems. The model has identifies nine factors as the main elements in the deterioration process of storm drainage systems. The order of the elements (size, trees, climate conditions, slope, soil, depth, location, structure, and age) is ranked according to the significance of their effect on the drainage systems. The 
research has merged management skills such risk analysis, as well technical skills such as pipe calculations to achieve the main aim of this study.

The framework of infrastructure risk analysis model shown in Figure 1 is developed by Ezell [3].
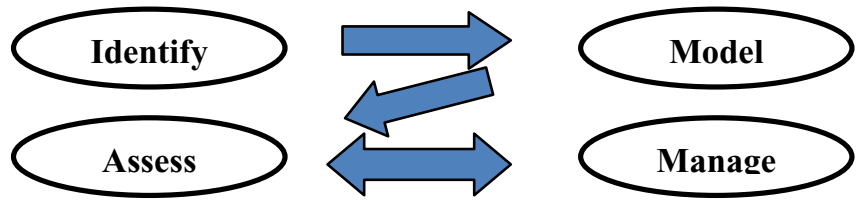

Figure 1: $\quad$ Infrastructure risk analysis model.

The model consists of four major phases, as described below:

i) Identify problems: The problem or the threat is identified through a case study at Southport, Queensland. The storm drainage pipe calculations, as well as the rainfall data, for the last 120 years (1887-2007) are collected.

ii) Model impact: Neural Network Deterioration Model (NNDM) is used to rank order the main factors that are responsible for the storm drainage deterioration.

iii) Assess risk: In this phase, the research applied risk analysis and management tools, such as Event Tree Analysis (ETA), Probability Density Function (PDF) and the Cumulative Distribution Function (CDF). These tools provide probability in percentages for the expected and extreme storm drainage damage.

iv) Manage infrastructure: After the expected, extreme damage probabilities are produced, the decision maker can investigate the level of risk or the precent of damage that is deemed to be acceptable. If the level of risk is not acceptable the model suggests going back to the third phase to reassess the situation and make adjustments where appropriate for new alternative solutions, for example: a new pipe sizing design, or an upgrade of the existing pipe diameters.

\section{Study area - Southport, Queensland, Australia}

Southport was one of the earliest established areas on the Gold Coast (Figure 2), it plays an important role as an administrative and business centre for the Gold Coast (GCCC [4]). Also, Gold Coast City Council (GCCC) has provided detailed information, such as topographic data, existing storm drainage, as well as maps for the designated flood affected areas in Southport. The study used the storm drainage underneath Bailey Court (as marked in red oval in Figure 2) as a sample investigation into the deterioration of the storm drainage. Most of the drainage for this area had been installed around 20 years ago. This particular location was chosen as that storm drainage exists next to one of the designated flood affected areas in Southport. The investigated location is situated next to the area seen as a threat from flooding resulting from the accumulating water after a heavy rainfall event of $300 \mathrm{~mm}$ per day (GCCC [5]). 


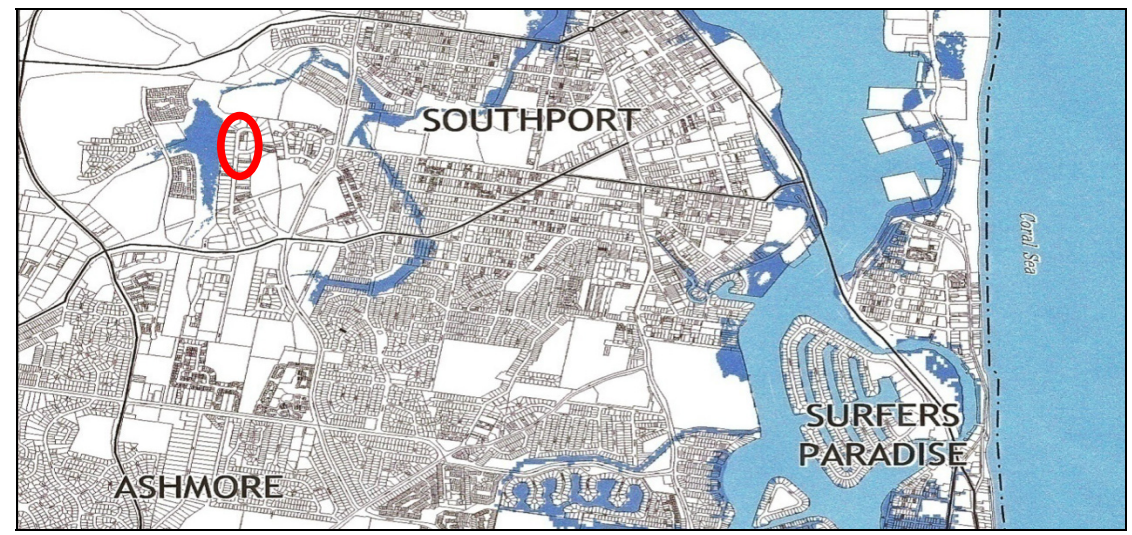

Figure 2: $\quad$ Designated flood affected areas in Southport, Queensland.

\subsection{Rainfall data analysis (1887-2007)}

The raw data of the daily rainfall for the Southport Ridgeway Avenue Station were provided by the Bureau of Meteorology (BOM), Brisbane Office BOM [2]. Figure 3 shows one sample period of 2005 to 2007.

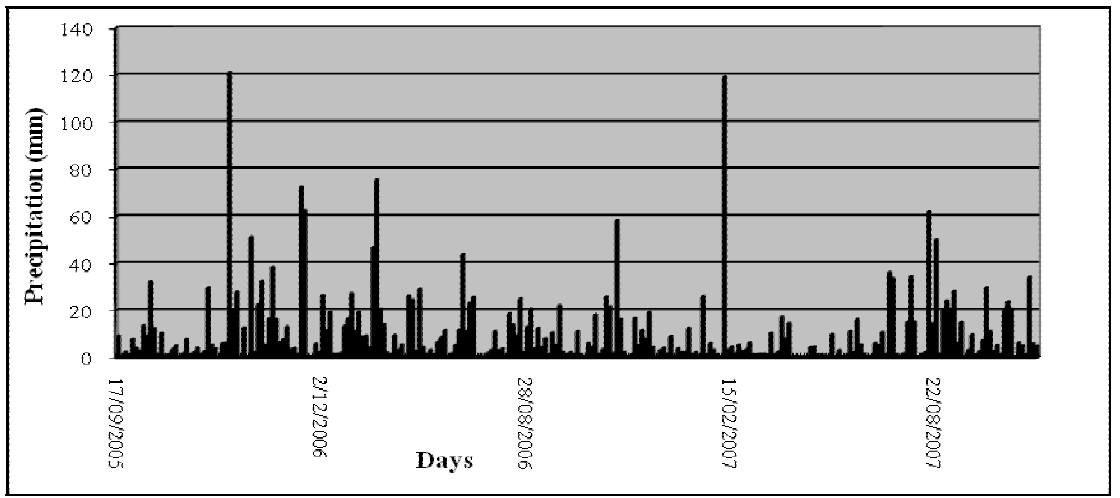

Figure 3: Daily rainfall rate for Southport Ridgeway Avenue Station, Queensland from 2005 to 2007.

After collecting the rainfall data, further investigation was undertaken through best fitting the entire data into a normal distribution. This analysis helped to provide the probability for the daily rain over $40 \mathrm{~mm}$. It is found that $13 \%$ of the data is above $40 \mathrm{~mm}$ per day. In addition, the threshold method was applied to determine the amount of data above the threshold of $40 \mathrm{~mm}$. This step was used to identify the behaviour or the trend of rainfall in the last 120 years. The technique allows for frequencies and patterns to be observed. The threshold, set at $40 \mathrm{~mm}$ per day, as in Figure 4(a). The rainfall above the threshold is presented in Figure 4(b). This Figure shows that Southport is getting less rain, through 


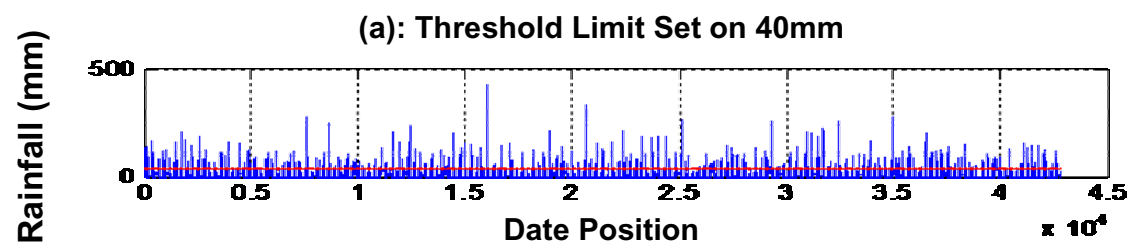

(b): Rainfall Data Detected Above Threshold

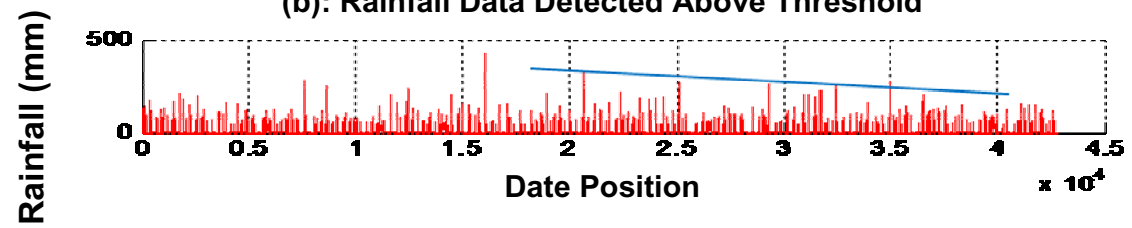

(c): Low-pass Filter and Rainfall Data Detected Above Threshold

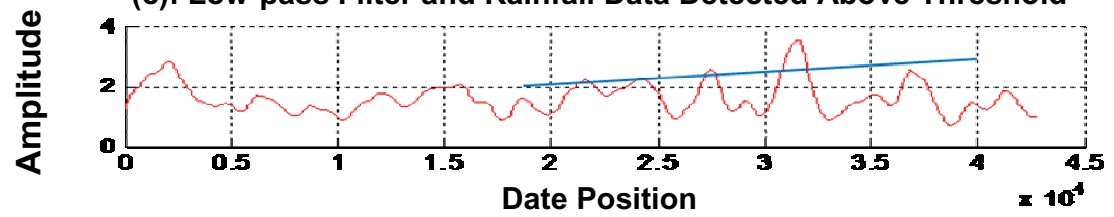

Figure 4: (a) The threshold line set on 40mm, (b) the rainfall data detected above the threshold and its decreasing trend, and (c) the amplitude of the rainfall frequency above the threshold, also it shows the increasing trend of frequency above threshold.

time. Figure 4(c) shows the frequencies or the concentration of the data identified. The trend of the chart is described as a unit-less trend, wave amplitude with a low-pass filter of 0.005 . It shows the frequency of the data in the second chart. Consequently, as observed in the third chart and represented by the drawn straight lined trend which connects the peaks in the chart (c), the rainfall patterns and frequencies are increasing through time despite the decrease in the total rainfall in chart (b).

\subsection{Rainfall probability estimation}

According to Alemi [1], the time to event method produces probabilities for a selected event through the examination of time in relation to the event. The method assumes that an event has a Bernoulli distribution (the event either happens or does not happen; It has a constant probability of occurrence, and the probability of the event does not depend on prior occurrences of the event). Thus the number of consecutive occurrences of the event has a Geometric distribution. In a geometric distribution, the probability of a rare event, $P$, can be estimated from the average time to the event, using the following formula (Alemi [1]):

$$
P=\frac{x}{y t}
$$


where, $\mathrm{P}$ is the probability of a specified event; $\mathrm{x}$ is the number of a specified event in a defined duration; $t$ is a defined duration; $y$ is the total number of events for a unit duration. The implementation of the formula above can be represented as in Figure 5.

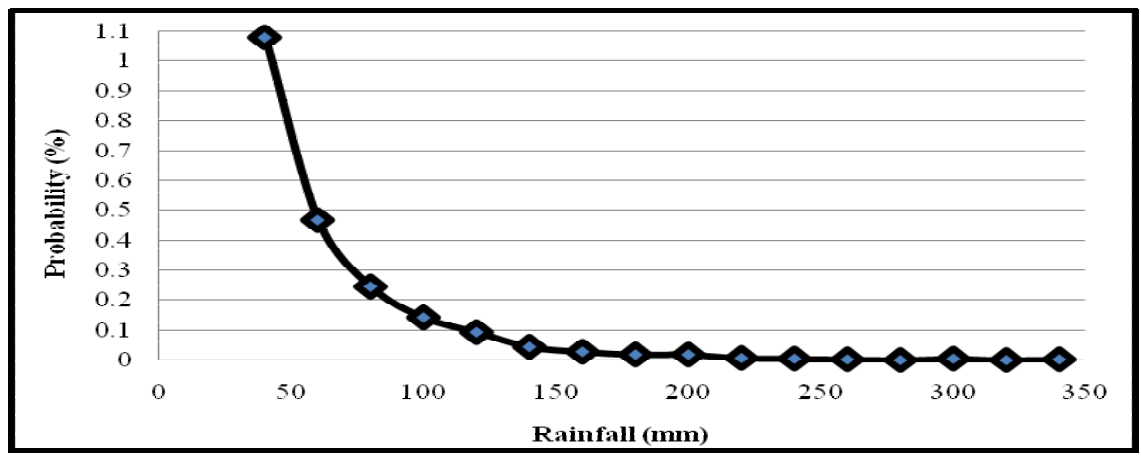

Figure 5: Probability percent of rainfall $(\mathrm{mm})$ for Southport, Queensland from 1887 to 2007.

\subsection{Runoff and pipe calculation}

The runoff and pipe flow are calculated to identify the rainfall conditions that experienced by the pipe at its full flow state. Any excess flow or derbies, such as tree branches and rocks that are carried by the flow, will increase the probability of pipe failure. The calculations relied on the flood estimation criteria of the Australian Rainfall and Runoff Guide (Pilgrim [6]). Additionally, while there are many methods used in Australia for flood estimation, this study has adopted the Main Roads Department Rational Method (RM) formula used in Queensland (Pilgrim [6]).

$$
Q_{r}=0.278 C_{Y} I_{t_{c}, Y} A
$$

where $Q_{r}$ is the peak flow rate $\left(\mathrm{m}^{3} / \mathrm{s}\right)$ of the Average Recurrence Interval (ARI) of $Y$ years; $C_{Y}$ is the runoff coefficient (dimensionless) for ARI of Y years; $I_{t c, ~}$ is the average rainfall intensity $(\mathrm{mm} / \mathrm{hr})$ for design duration of $\mathrm{t}_{\mathrm{c}}$ hours and ARI years; $\mathrm{A}$ is the area of catchment $\left(\mathrm{km}^{2}\right)$. The value of 0.278 is the conversion factor to balance the units.

Eqn. (2) is set to determine the critical full flow point for the pipe; essential values need to be derived so that they can be substituted into the RM formula, such as the area of the catchment, the velocity of the runoff and the coefficient of the runoff. The Catchment area was determined by a site visit, and topographic data for the Bailey Court Location provided by Geoscience Australia. In addition, The Queensland Department of Main Roads RM has determined runoff velocities for the time of concentration $\left(t_{c}\right)$ for areas less than $5 \mathrm{~km}^{2}$, Moreover, for the determination of the runoff coefficient, four values need to be identified (Rainfall intensity, relief, storage and ground characteristics and cover) Pilgrim [6]. Table 1 shows the results deduced form eqn. (2). The corresponding pipe 
diameter to $\mathrm{Q}_{50}$ calculated is $\mathrm{D}=1200 \mathrm{~mm}$. Yet, the existing storm sewer pipe diameter is $900 \mathrm{~mm}$. So, there will be new value for $\mathrm{I}_{\mathrm{tc}=0.2 \mathrm{hr}, \mathrm{Y}=50 \mathrm{yr}} \approx 40 \mathrm{~mm} / \mathrm{hr}$. That means, with a rainfall rate of $40 \mathrm{~mm} / \mathrm{hr}$, the storm sewer will experience a full flow state.

Table 1: $\quad$ Calculated values for eqn. (2).

\begin{tabular}{|c|c|c|c|c|c|}
\hline $\begin{array}{c}\text { Area } \\
\left(\mathrm{km}^{2}\right)\end{array}$ & $\mathrm{T}_{\mathrm{c}}(\mathrm{hr})$ & $\mathrm{Y}(\mathrm{yrs})$ & $\mathrm{I}_{\mathrm{tc}, \mathrm{Y}}(\mathrm{mm} / \mathrm{hr})$ & $\mathrm{C}_{50}$ & $\mathrm{Q}_{50}\left(\mathrm{~m}^{3} / \mathrm{s}\right)$ \\
\hline 0.144 & 0.20 & 50 & 200 & 0.85 & 6.8 \\
\hline
\end{tabular}

\section{Risk analysis tools}

There are many risk analysis and management tools that can quantify a risk both objectively and subjectively. The tools adopted in the research were based on the induction and empirical observation of historical rainfall data for the last 120 years in Southport. The impacts will be quantified by using the Event Tree Analysis (ETA) tool, followed by the generation of the Probability Density Function (PDF) and the Cumulative Distribution Function (CDF) tools. As same tools require a rank order for the impacted factors, the Neural Network Deterioration Model was used to provide the ranking order of factors that contributes to the deterioration of storm water drainage, is used. The model and the risk analysis and management tools are detailed in the subsequent sections.

\subsection{Event tree analysis}

The consequences of the event are followed through a series of possible paths. Each path is assigned a probability of occurrence, so that the probability of the various possible outcomes can be calculated. The damaging ranges were assumed to be uniform and categorized into small from 0 to $5 \%$, medium from 5 to $25 \%$ and large from 25 to $50 \%$. It was decided to measure the damage to $50 \%$, as any damage more than $50 \%$ will result in near total failure for the storm drainage pipe. Neural Network Deterioration Model (NNDM), developed by Tran et al. [7], specializes in identifying and ranking the deterioration factors of storm drainage. NNDM has been determined that there are nine factors that have damaging effects to storm drainage. The model developed a ranking order for the deterioration factors - the factors are size, trees, climate conditions, slope, soil, depth, location, structure and age - which are ranked from 1 being the most important to 9 being the least important (Tran et al. [7]). Moreover, the model stated that two factors (age and structure) had an insignificant effect when comparing them with the other factors. Consequently, the research adopted factors one to seven in the analysis.

The ETA was implemented by setting the seven ranked factors from the NNDM sequent events, and inserting the first factor (size) to have the highest weight value. The factors were weighted, respectively, according to their ranking order in the $\operatorname{NNDM}(50,40,30,20,10$ and 1\%), as shown in Figure 6. However, this research concentrated on the factor of climate conditions. 


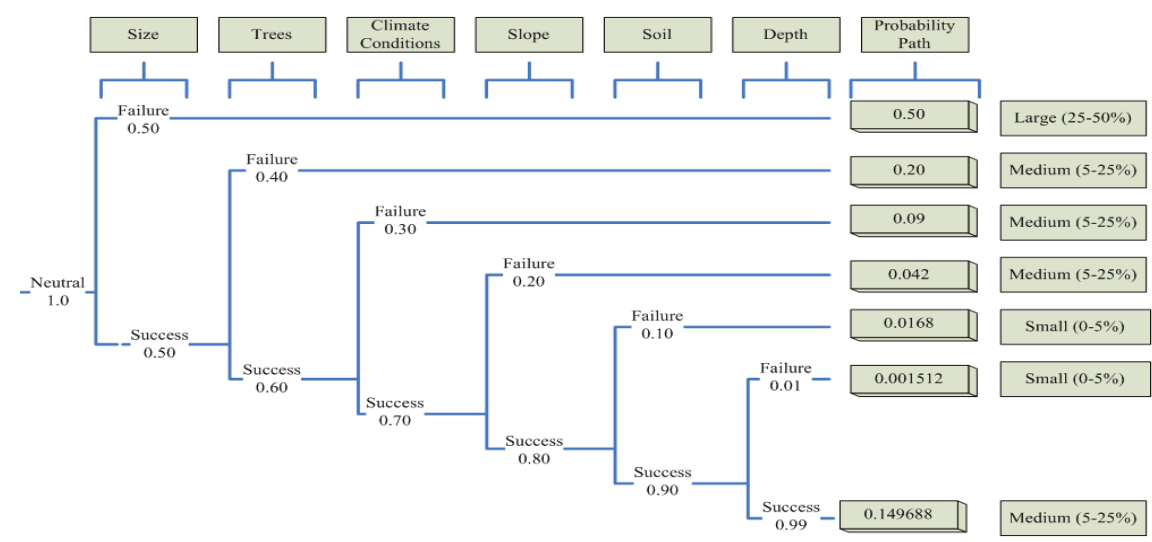

Figure 6: Implemented event tree analysis according to NNDM.

To examine the $\mathrm{CC}$ impact, three scenarios were assumed to observe the behaviour of the rainfall and storm drainage relationship. The scenarios were constructed to imitate, or imply the extreme conditions they might be expected under CC. In other words, the factor of climate conditions will be the centre of the analysis; by building three scenarios where all factors will be fixed, in terms of weighting value, then the climate conditions factor will be increased. The scenarios were established on the basis of the incrementing climate conditions factor, while all other factors were fixed. The first event tree analysis shows the normal situation of the NNDM, where all factors were weighted according to their ranking order in the NNDM. The normal and unchanged situation was generated as a benchmark for the following scenarios, specifically in terms of analysis and behaviour observation

\subsection{Probability density function}

PDF demonstrates the ETA outcomes, or the probability path of the event. Further, the distribution for the outcomes from the ETA in the normal situation was assumed to be uniform in three ranges (small, medium and large damages). The probability path is produced in ranges from small to large. These ranges are then plotted in a chart as the x-axis; the corresponding probability paths are plotted as the y-axis. Figure 7 illustrates the implementation of the events depicted in Figure 6. After depicting the normal situation, all the other factors were fixed, while the climate conditions factor was increased: $10 \%$ (from 30 to $40 \%$ ) for scenario 1 ; another $10 \%$ (from 40 to $50 \%$ ) for scenario 2 ; and, for the extreme event, $50 \%$ (from 50 to $100 \%$ ) for scenario 3. This scenario has the most damaging effect on the drainage system. Interestingly, for scenario 3, the small and medium damaging ranges have become lower, while the large damaging range has become larger, when compared with scenario 1 or the normal situation. 


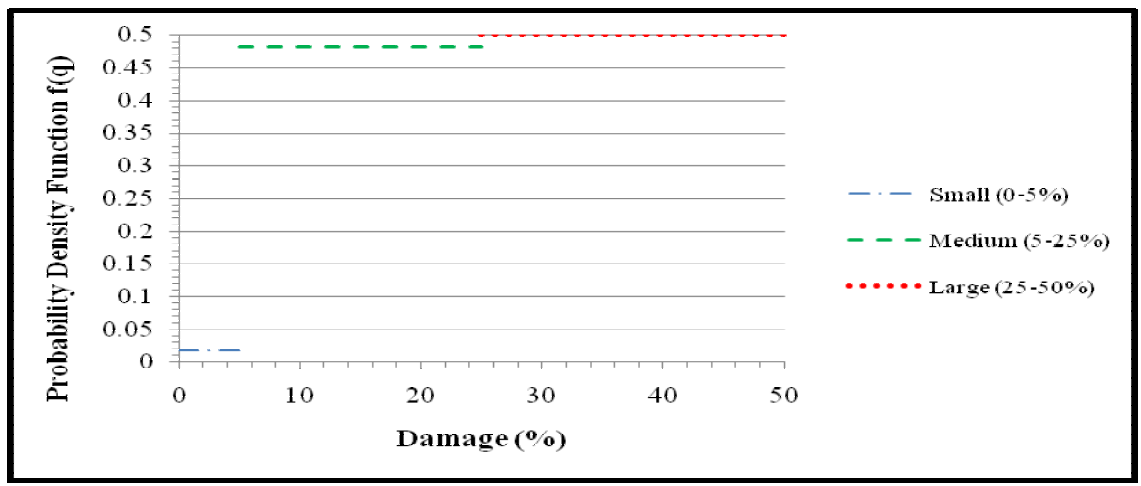

Figure 7: Probability density function for the normal situation of the NNDM.

\subsection{Cumulative distribution Function}

CDF can be used to better identify the behaviour of the area under the PDF. It provides a combined comparison between all the scenarios in a single chart, as shown in Figure 8 . By increasing the weight value of the climate condition factors in each scenario, the slope of the small damaging range is becoming lower, and the slope of the medium is becoming larger.

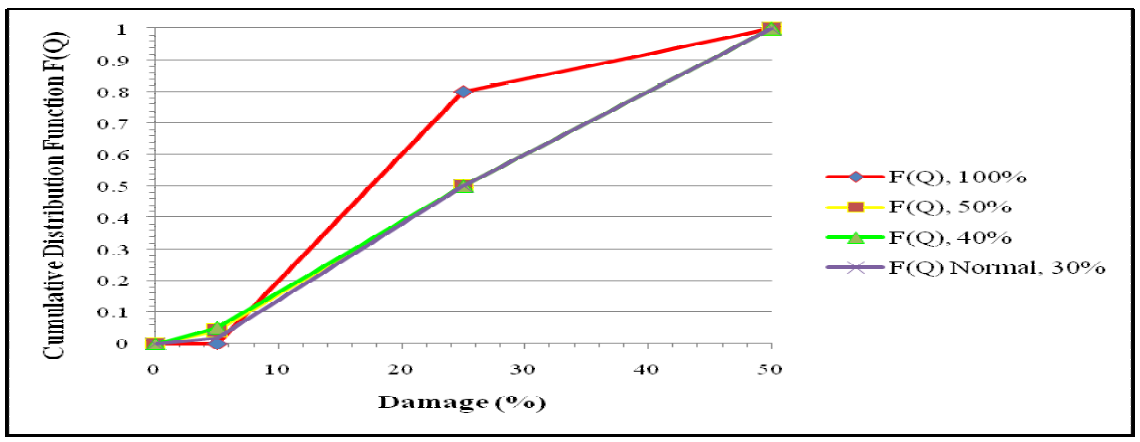

Figure 8: $\quad$ Combined CDF chart for all scenarios.

\subsection{Relationship between rainfall and pipe damage}

The relationship between the rainfall and the damage percentage of storm drainage are shown in Figure 9. A range of rainfall from $40 \mathrm{~mm}$ to $300 \mathrm{~mm}$ is given. This assumption was based on the failure critical point, as defined in the pipe calculation section, as $40 \mathrm{~mm} / \mathrm{hr}$. This critical point was placed at the lower range of the assumption, being $0 \%$ damage.

According to official records from the GCCC, a rainfall event of $300 \mathrm{~mm}$ per day would result in a total damage made by flood for Gold Coast city (GCCC [5]). Consequently, $300 \mathrm{~mm}$ per day was placed at the highest range of the 


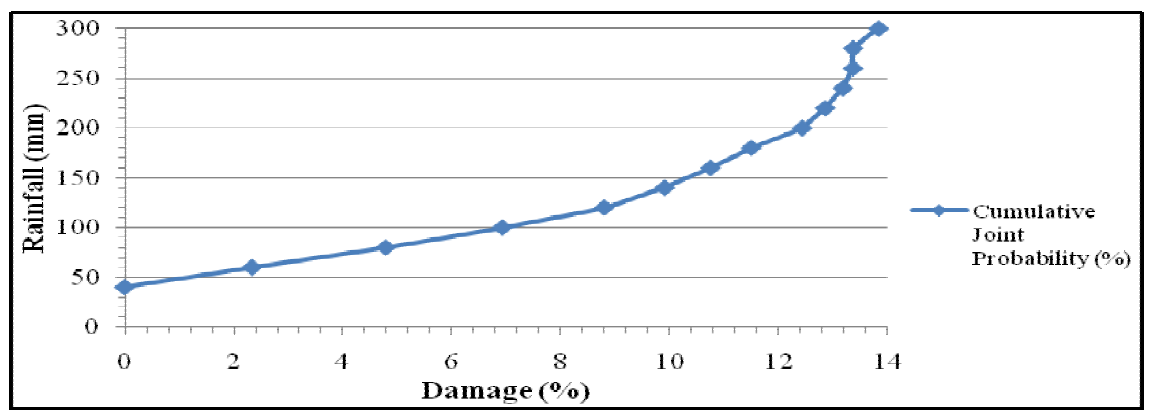

Figure 9: Combined probabilities between rainfall in $\mathrm{mm}$ and storm drainage damage in percentage.

assumption, which is $100 \%$ damage. The assumed range was also mirrored in parallel with the rainfall probability that was generated in Figure 5. The outcome from the combined probabilities between the rainfall, in $\mathrm{mm}$, and the cumulative storm drainage, damage in percentage is presented in Figure 9.

\subsection{Risk quantification}

The expected and extreme vulnerability of pipes to rainfall impact was quantified using statistical risk analysis and management quantification tools, namely ETA, PDF and CDF, to quantify the risk of failure. The risk quantification of the rainfall damage to storm drainage is presented as probability percentages. The probabilities produced give important data, and their implications, for both engineers and decision makers. It would be wise for engineers to take these damage probabilities into account when designing safety factors for storm drainage design. This is especially so, with the increasingly litigious society in Australia.

While decision makers may accept a risk under certain conditions, in accordance with their management strategies, sometimes they will deem the risk as unacceptable. This study has provided the means for adjusting or reassessing the risk, to ensure that the most feasible level of risk is attained. In other words, decision makers can set damage probabilities as benchmarks for both the most safe and most economical outcomes.

\section{Conclusion}

The challenges, considerations, responsibilities, and processes of engineers in coping with CCs have been identified by this research. The responsibilities of engineers are growing in terms of providing appropriate and adequate infrastructure engineering. Innovative design and development will ensure that infrastructure failure is minimised or reduced.

Climate change is one of the most important global issues facing the world today. These changes are affecting engineering infrastructure, one of the most vital sectors to human life and work. Storm drainage is an important part of that 
engineering infrastructure. Indeed, it is the first defence line in protecting urbanized cities from the risk of flood and inundation. For this reason city councils and local authorities need to pay greater attention to this essential infrastructure sector. For example, storm drainage exists under roads, and so when storm drainage failure occurs the road infrastructure will be at risk. In other instances it could be bridges and building foundations at risk. As a consequence both public and private sector costs rise. The fundamental importance of engineering infrastructure to daily life motivated the author to investigate the impacts that rainfall, extreme rainfall, may have on this sector. For this reason, the objective of this research was to quantify the impacts of rainfall on storm drainage resulting from climate change. The main results and findings of this study are discussed in the following sections.

This research examined CC impacts on engineering infrastructure design and planning on the Gold Coast. It applied risk analysis and management techniques to identify how storm drainage could adequately cope with the expected CC impacts. In addition, the study also addressed the adaptation guidelines for architects and engineers to assist with appropriate infrastructure designs.

The findings show that rainfall trends, patterns and frequencies have changed in the last 120 years for the Southport area. Further, such differences are expected to increase in the future, depending on the $\mathrm{CC}$ trends and their level of significance. Also, a rainfall event, resulting from $\mathrm{CC}$, of more than $40 \mathrm{~mm} / \mathrm{hr}$ in Bailey Court, Southport, will increase the probability of storm water drainage damage. The critical failure point was identified by this research; also the storm water drainage damage has been quantified in probability percentages. The probabilities, generated by using the risk analysis and management measurement tools, provided valuable observations of the expected storm water drainage behaviour under a certain rainfall event. These findings will help decision makers and engineers to choose acceptable risks from certain rainfall events or adapt better strategies to tackle such impacts.

\section{References}

[1] Alemi F. (1996, 27 January, 2008). Decision Analysis and Measuring Uncertainties.Available:

http:/gunston.doit.gmu.edu/healthscience/730/ProbabilityRareEvent.asp\#Pr obabilityofRareEvents

[2] BOM (Buere Of Meterology). (05 March, 2008). Design Rainfall Intensity Chart. Available: http://www.bom.gov.au

[3] Ezell B. C.. Infrastructure Risk Analysis Model (IRAM), Journal of Infrastructure Systems, vol. 27, 2000.

[4] GCCC (Gold Coast City Council). (2008, Heritage Towns. Available: http://www.goldcoast.qld.gov.au/

[5] GCCC (Gold Coast City Council). (2003, November 04, 2007). Important Information. Available: http://www.goldcoast.qld.gov.au 
24 Flood Recovery, Innovation and Response II

[6] Pilgrim D. H., Australian rainfall and runoff : a guide to flood estimation. Vol. 1, Reprinted ed. Barton, A.C.T.: Institution Of Engineers, Australia, 2001.

[7] Tran D. H., et al., Neural networks deterioration models for serviceability condition of buried stormwater pipes, Engineering Applications of Artificial Intelligence, vol. 20, pp. 1144-1151, 2007. 